\title{
CONSOLIDAÇÃO DE MATERIAL PÉTREO POR MEIO DO PROCESSO
}

\section{SOL-GEL HÍBRIDO}

\author{
Januaceli Felizardo Murta ${ }^{1}$, João Cura D'Ars de Figueiredo Júnior ${ }^{2}$, \\ Marco Antônio Penido de Rezende ${ }^{3}$
}

1 - Arquiteta Urbanista; 2 - UFMG. Dep. de Artes Plásticas - EBA; 3 - UFMG. Dep. de Tecnologia da Arquitetura e Urbanismo - EAU janumurta@gmail.com

\begin{abstract}
Resumo: A rocha é uma das matérias mais antigas utilizadas nas construções, na qual a arte de esculpi-las é tida também como uma das mais antigas habilidades do ser humano. Apesar de tais indícios, este material é um dos mais recentes a ser enfocado na ciência da conservação, e ainda com soluções incipientes. A partir do exposto, a pesquisa realizada até o momento buscou conhecer as patologias recorrentes dos materiais pétreos, e os métodos existentes de diagnóstico, intervenção e testes (destrutivos e não destrutivos) focados na conservação das peças em tal material. Só após este levantamento prévio procedeu-se a elaboração de produto para a consolidação da pedra sabão (processo de injetar no material danificado um novo material que preencha as cavidades internas, fornecendo melhores propriedades mecânicas à obra): o uso do processo sol gel - anteriormente utilizado, apresentou problemas de deterioração em muitos bens culturais como aceleração do processo de degradação, bem como alteração cromática. A estrutura híbrida dos alcóxidos com siliconas - o polidemetilsiloxano (PDMS) - foi testada com resposta eficiente, na qual ocorreu melhoria das características mecânicas e hidrofobicidade dos fac-símiles.
\end{abstract}

Palavras Chave: consolidação de material pétreo, processo sol gel, Sol-gel híbrido, TEOS, PDMS, pedra sabão

Abstract: CONSOLIDATION OF STONE MATERIAL BY HYBRID SOL-GEL PROCESS. The rock is one of the oldest materials used in construction, in which the art of sculpting it is considered also as one of the oldest skills of human beings. Despite such evidence, this material is one of the latest to be focused on conservation science, and with incipient solutions. From the foregoing, the research conducted to date sought to meet the recurrent pathologies of stone materials, and the existing methods of diagnosis and intervention trials (destructive and nondestructive) focused on maintenance of parts such material. Only after this preliminary survey it proceeded to the preparation of product for the consolidation of the soapstone: the use of sol gel process - previous use showed deterioration problems in many cultural assets as accelerating the degradation process and color change. Now, this hybrid structure of alkoxides with silicones - the polidemetilsiloxano (PDMS) - was tested with efficient response, as improved mechanical properties due to reduced porosity and hydrophobicity as the reduction of water absorption of the facsimiles.

Keywords: consolidation of stone material, sol gel process, hybrid sol-gel, TEOS. PDMS, soapstone

\section{INTRODUÇÃO}

A rocha é uma das matérias mais antigas utilizadas nas construções, na qual a arte de esculpilas é tida também como uma das mais antigas habilidades do ser humano. (COSTA, 2009). Apesar de tais indícios, este material é um dos mais recentes a ser enfocado na ciência da conservação, e ainda com soluções incipientes.

Entre o conjunto de esculturas e de outros objetos de arte em Minas Gerais até fins do século XIX, o maior número de obras é em pedra talco ou em pedra sabão (COSTA, 2009), com destaque para as obras do mestre Aleijadinho (Antônio Francisco Lisboa; 1738-1814), sendo as imagens em pedra sabão do santuário em Congonhas do Campo (17951807) uma das principais obras. Vale ressaltar que os blocos utilizados na Vila Rica do século XVIII eram avulsos ou facilmente extraídos e, a partir desse, selecionavam o mais acessível e econômico. Não existem registros históricos acerca das antigas áreas de extração em Minas Gerais, estando exauridas as possíveis áreas extrativas, ou estarem locadas em atuais áreas de preservação ambiental. (SILVA, 2007). Especificamente em relação ao conjunto escultural dos doze profetas de Congonhas do
Campo ${ }^{1}$, Costa (2009) revela que a pedra sabão foi extraída das redondezas, apresentando significativa heterogeneidade de composição, cor e textura.

Em relação à deterioração dos materiais pétreos, Angeli (2007) informa que essa é muito semelhante à que acontece na natureza, ou seja, nos afloramentos da rocha. Entre as causas de alteração, estão os parâmetros ambientais, como chuva, vento, diferenças de temperatura, e presença de sais solúveis; e de origem antrópica, como existência de poluentes atmosféricos ${ }^{2}$ e restauração sem critério (realizada de maneira errada). Uma das principais causas de deterioração (IPHAN, 2000; ANGELI, 2007) é a presença de sais solúveis que geram alterações químicas e mecânicas - a entrada por capilaridade de soluções salinas, com cristalização e recristalização de sais e quebra interna que são lixiviados pelo movimento natural de entrada e saída de água (evaporação, alterações climáticas, intempéries). Segundo Figueiredo Júnior (2012), a recristalização pode gerar pressões de até 1.000 atm (aproximadamente $100 \mathrm{Mpa}$ ). Um sal comum

${ }^{1}$ Embora o santuário já estivesse concluído em 1777, os profetas foram confeccionados entre os anos de 1800 e 1805. O conjunto é tombado pelo IPHAN (SPHAN,1939) e reconhecido pela UNESCO como Patrimônio Mundial (1985). (ROBERT, [200-]). ${ }^{2}$ Storemyr (2004) relata o impacto das alterações climáticas na pedra, citando o aquecimento observado no século XIX devido ao uso expressivo do carvão como fonte de energia; e posteriormente com o desenvolvimento industrial, crescimento populacional e consequente aumento da poluição. 
responsável pela deterioração no decaimento salino é o sulfato de sódio, $\mathrm{Na}_{2} \mathrm{SO}_{4}$, também conhecido com ternardita-a conversão para mirabolita, sua forma hidratada, pode gerar pressões de 254,23 atm (aproximadamente $26 \mathrm{Mpa}$ ). (ESPINOSA, 2008).

No ano de 1941, a pedido do SPHAN, Orosco fez um estudo das avarias já que haviam desgastes que geravam o temor da destruição pela ação do tempo. (Revista do SPHAN, 1941).À época foi constatado que o abandono (ou a ausência de ações de conservação e manutenção) gerava a mutilação das peças por ações de vandalismo (FIGURA 1), observando ainda que a população não respeitou o conteúdo artístico-estético da escultura, tampouco o significado religioso. (OROSCO, 1941). (FIGURA 2).

Por fim Orosco (1941) conclui que não serão os elementos naturais responsáveis pelas avarias, sendo a erosão pouco significativa, uma vez que há trechos intactos ladeados por partes avariadas. Como cuidados, é listado, entre outras, a conservação da pátina que tem papel consolidador das partes alteradas, além de oculta-las.

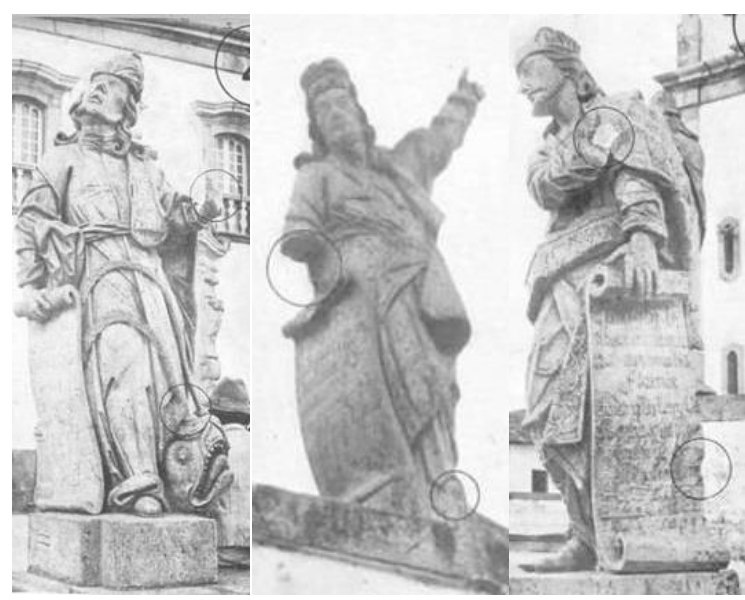

Figura 1. Mutilações sofridas pelos profetas: dedos de Jonas; antebraço e pé de Habacuc; mão, punho e painel de Ezequiel (OROSCO, 1941, p. 181, 182)

A visão apresentada por Orosco (1941) é contraposta por Herkenrath et al. (1994 - IDEAS Project ${ }^{3}$ ) que afirma que há uma aceleração da deterioração relacionados aos novos mecanismos de intemperismo ${ }^{4}$ conhecidos até o momento, que por sua vez estão interligados à poluição do ar com aumento das substâncias antrópicas, além de outros fatores, exigindo a pesquisa de medidas para a

3 IDEAS Project é uma parceria Brasil-Alemanha, visando adaptar a metodologia existente para pesquisa e avaliação de processos de deterioração da pedra.(HERKENRATH et al., 1994, p. 19).

4 Intemperismo seria o processo natural relacionado à formação dos solos, considerado lento e pouco perceptivel em termos de um ciclo de vida humana podendo, entretanto ser visivel em monumentos, já que este use mostra acelerado e visivel em poucos anos. (HERKENRATH et al., 1994, p. 18). proteção da pedra. A cristalização e recristalização de sais podem ainda ser consideradas o fator mais importante de aceleração da degradação da pedra pela ação de poluentes atmosféricos, já que possuem alto poder de destruição. Em Congonhas o principal influenciador da qualidade do ar é o intenso tráfego de veículos que, com a chuva, contribui ainda mais para o processo de deterioração. A umidade, principal fator que contribui para a geração de danos, deve ser o principal elemento a ser controlado nas estruturas em material pétreo. (HERKENRATH et al., 1994).

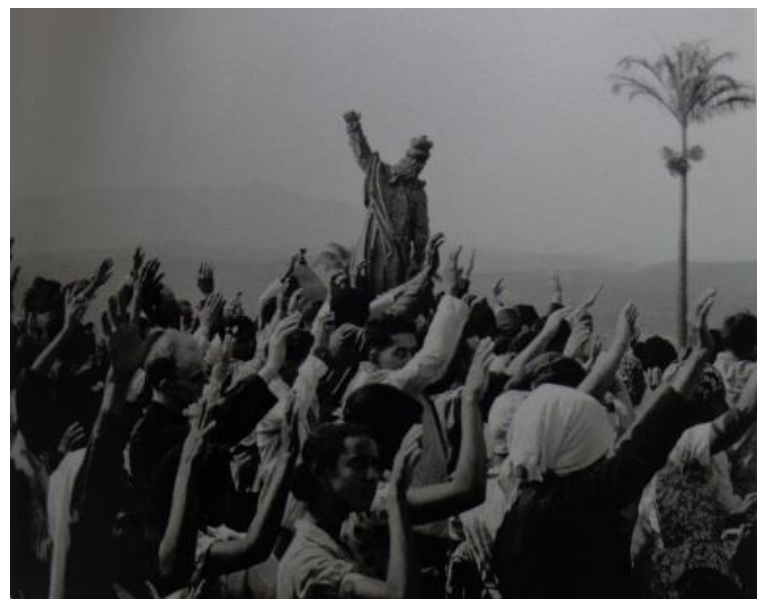

Figura 2. Romaria do Jubileu do Senhor Bom Jesus de Matosinhos, 1950 (CARMO, 1973)

Sem modelo de degradação válido para todas as condições e tipos de pedras, nenhum agente de conservação pode ser especificado ${ }^{5}$, no qual foi indicado o estudo de material adequado para reduzir fissuras e danos por intemperismo nas peças (consolidantes) $^{6,}$ bem como de material para reduzir a umidade superficial, necessitando de testes a longo prazo com exposição dos exemplares nas mesmas condições das originais, com acompanhamento do processo de degradação, eficiência e durabilidade das técnicas e materiais de restauro. (HERKENRATH et al., 1994.).

Por meio de um levantamento fotográfico, apesar das esculturas mostrarem-se íntegras quando avaliadas em conjunto, é nítido que há perdas consideráveis que começam a comprometer a integridade de partes específicas de cada uma das peças: (FIGURA 3 a FIGURA 5).

5 A limpeza e aplicação de biocidas são realizadas periodicamente há alguns anos, mas o período de reincidência tem sido cada vez menor. Novos produtos devem se pesquisados para tal fim, bem como as consequências que tais aplicações podem ter no futuro tanto para a pedra, como para o entorno próximo. A dessalinização pode também ser um dos processos que contribuam para a conservação do conjunto.

6 Alguns materiais a base de éster de ácido silícico e pó de pedra foram testados, 6 Alguns materiais a base de éster de ácido silcico e pó de pedra foram testados, (HERKENRATH et al, 1994). 


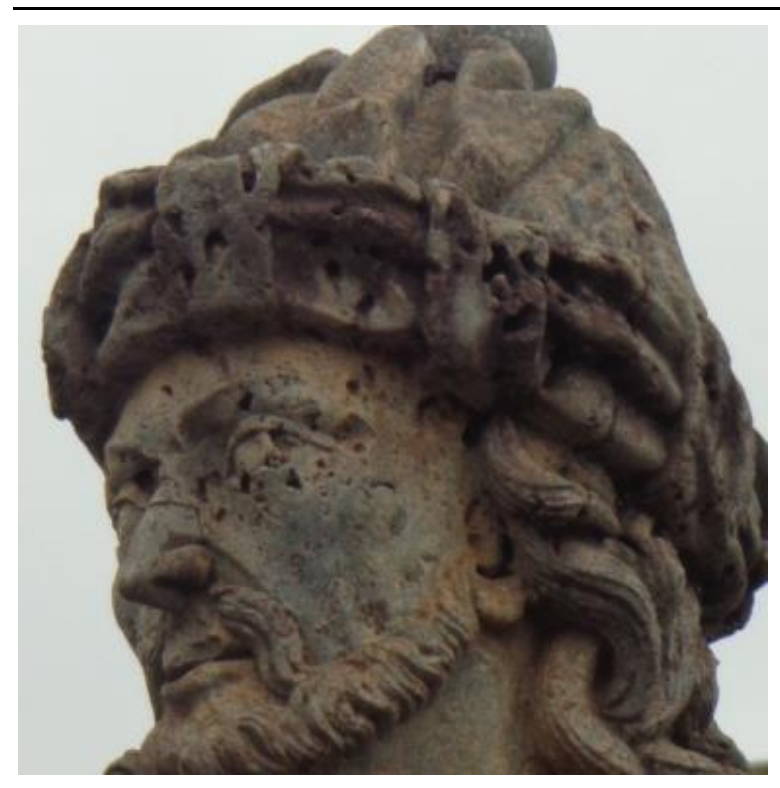

Figura 3. Danos verificados no Profeta Jeremias (autores, 2014)

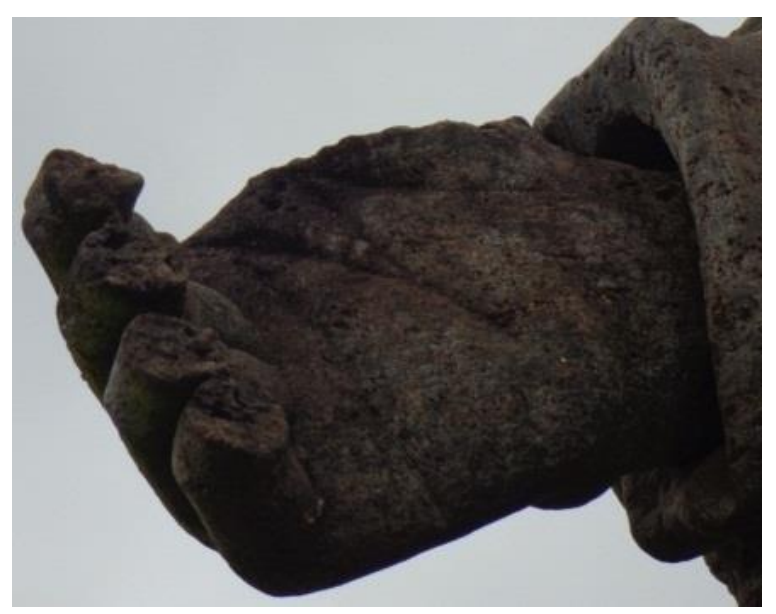

Figura 4. Danos verificados no Profeta Amós (autores, 2014)

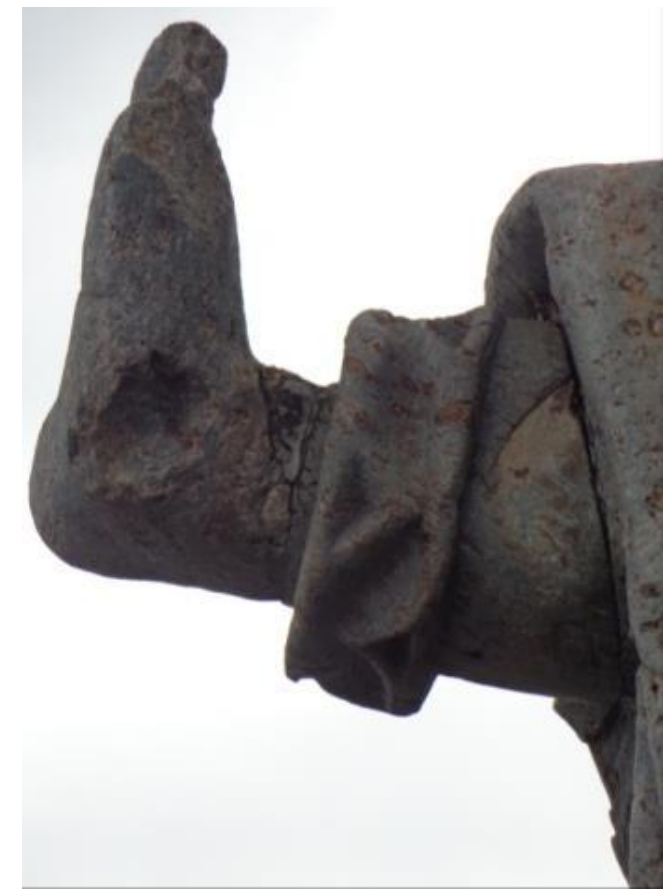

Figura 5. Danos verificados no Profeta Jonas (autores, 2014)

\section{CONSOLIDAÇÃO}

Consolidação é a "impregnação de produtos que penetram na pedra, melhorando e aumentando a coesão do material alterado em seu substrato, resultando na melhor resistência aos processos de deterioração". (IPHAN, 2000, p. 35.). ${ }^{7}$ A priori, os consolidantes:

[...] não devem formar subprodutos deteriorantes; devem ser absorvidos uniformemente pela pedra; terão a profundidade de penetração controlada; devem ter o coeficiente de dilatação térmica próximo do da pedra a ser consolidada; não devem tornar a pedra totalmente impermeável; devem manter a aparência externa da pedra. (IPHAN, 2000, p. 35).

O ideal seria aplicar tratamentos reversíveis, mas, no contexto da pedra, essa possibilidade é mais idealista do que realista. É sensato, portanto, assumir que o produto não poderá ser removido ${ }^{8} \mathrm{e}$, frente a esse fato, gerações futuras terão de conviver com as consequências das ações atuais. Mas se por um lado o tratamento é irreversível, a negligência também o é devendo-se para tanto encontrar uma decisão de forma consciente, equilibrada e respeitosa. (DOEHNE; PRICE, 2010).

$\mathrm{O}$ tetraetilortosilicato [TEOS - $\mathrm{Si}\left(\mathrm{OC}_{2} \mathrm{H}_{5}\right)_{4}$ ] processo sol-gel - é usado há muito tempo para a consolidação de material pétreo. (HENCH; WEST, 1989; WHEELER, 2005). Entretanto, muitos bens apresentaram problemas de deterioração avançados devido ao próprio processo. A solução proposta é a adição de polidimetilsiloxano [PDMS - $\left(\mathrm{C}_{2} \mathrm{H}_{6} \mathrm{OSi}\right)_{n}$ ]. (FIGURA 6). Entre as vantagens, cita-se: 'homogeneidade' entre os compostos; flexibilidade; boa penetrabilidade; composto comercialmente disponível; e a alteração estética pode ser evitada. (ZÁRRAGA et al., 2009).

\subsection{Fase experimental}

O presente trabalho privilegia a aplicação do consolidante em pedra sabão, no qual pretende-se mostrar a aplicabilidade do processo em condições únicas do acervo mineiro. A fase experimental pode ser dividida em oito fases: desgaste artificial das amostras de esteatito (determinação da resistência à cristalização de sais); determinação dos índices físicos (determinação da densidade aparente, porosidade aparente e da absorção de água); determinação da resistência à compressão uniaxial; determinação da velocidade de propagação de onda ultrassônica; determinação de absorção de água por capilaridade; teste de colorimetria e o processo de

${ }^{7}$ Figueiredo Júnior (CURSO DE CARACTERIZAÇÃO E CONSERVAÇÃO DA PEDRA, 2014), cita como principais substâncias usadas como consolidantes o Hidróxido de Cálcio $\mathrm{Ca}(\mathrm{OH})_{2}$-, Hidróxido de Bário - $\mathrm{Ba}(\mathrm{OH})_{2}-, \mathrm{TiO}_{2}$ como catalisador, Bifosfato de amônio $\left(\mathrm{NH}_{4}\right)_{2} \mathrm{HPO}_{4}-$, Alcoxisilanos e Acrílicos.

$\left.{ }_{8}^{(} \mathrm{NH}_{4}\right)_{2} \mathrm{HPO}_{4}$ de se levar em conta que provavelmente a peça já tenha passado por intervenções anteriores, já estando impregnada por produtos que, muitas vezes, é desconhecido. 
desenvolvimento e caracterização do composto híbrido. Todos os testes aqui descritos são normatizados, com utilização da ABNT (Associação Brasileira de Normas Técnicas), ASTM (American Society for Testing and Materials), e NP (Normas Portuguesas). (Erro! Fonte de referência não encontrada.).

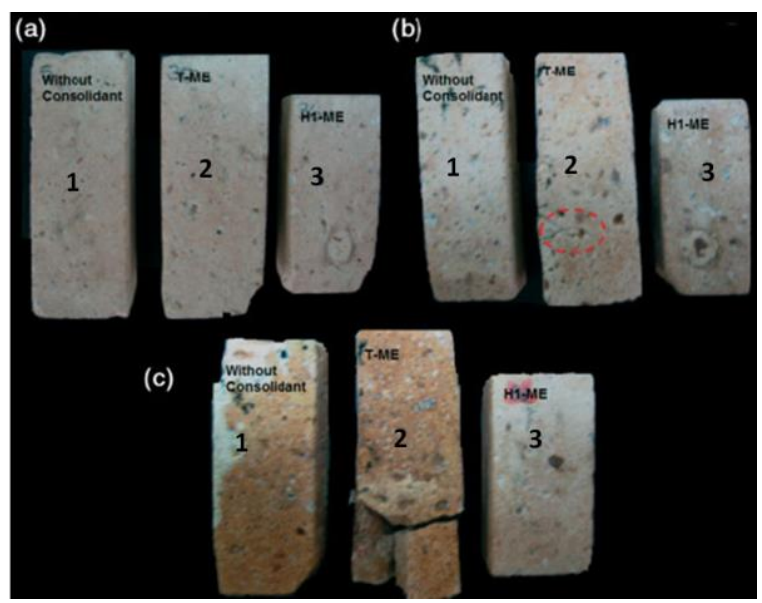

Figura 6. Tufa calcária (1), consolidada com TEOS (2) e com TEOSPDMS-sílica (3), sendo (a) as amostras, (b) após 5 ciclos de desgaste artificial, e (c) após 6 ciclos de desgaste artificial (SALAZAR-HERNANDÈZ et al., 2010, p. 487)

Tabela 1. Normas vigentes para embasamento da fase experimental (Elaboração dos autores)

\begin{tabular}{ll}
\hline ENSAIOS & Norma ABNT \\
\hline Análise Petrográfica & ABNT/NBR 15.845/10 - ASTM C-295 \\
\hline $\begin{array}{l}\text { Índices Físicos (determinação } \\
\text { da densidade aparente, da } \\
\text { porosidade aparente, e da } \\
\text { absorção de água) }\end{array}$ & ABNT/NBR 15.845/10 - ASTM C-97 \\
\hline $\begin{array}{l}\text { Resistência à Compressão } \\
\text { Uniaxial }\end{array}$ & ABNT/NBR 15.845/10-ASTM D-2.938/C-10 \\
$\begin{array}{l}\text { Determinação da velocidade } \\
\text { de propagação de onda } \\
\text { ultrassônica }\end{array}$ & ASTM D-2.845 - EN 12.504-4 (2004) \\
$\begin{array}{l}\text { Determinação da resistência } \\
\text { à cristalização de sais }\end{array}$ & NP EN 12.370 (2001) \\
$\begin{array}{l}\text { Determinação de absorção } \\
\text { de água por capilaridade }\end{array}$ & ABNT/NBR 9.779/1994 \\
\hline
\end{tabular}

As amostras foram adquiridas na pedreira WS Soapstone locada em Cachoeira do Campo, distrito do município de Ouro Preto. De acordo com análise petrográfica (LABTECRochas - IGC/UFMG, 2009), a rocha denominada comercialmente como 'Barroca' é constituída basicamente por talco, clorita, carbonato e óxidos na forma de cristais dispersos.

Para a obtenção de amostras minimamente deterioradas para posterior consolidação, foi utilizada a NP EN 12370/2001, que orienta o ensaio de determinação da resistência à cristalização de sais. A norma recomenda a execução de 15 ciclos da imersão dos provetes em solução de sulfato de sódio $\left(\mathrm{Na}_{2} \mathrm{SO}_{4}\right)^{9}$, com secagem em estufa (temperatura de $105 \pm 5^{\circ} \mathrm{C}$ ), até a perda de massa ou ruptura - é importante a dessalinização das peças, visto que o material fraturado pode continuar no interior da amostra.

A produção do produto em exposição foi embasada em artigos relacionados às recentes pesquisas acerca do composto híbrido(KIM et al, 2008; ZARRAGA et al, 2009; SALAZAR-HERNANDEZ et al, 2010; Ll et al, 2012; ZHAO et al, 2013) e, visando torná-lo comercialmente acessível, optou-se por extrair PDMS de um perfil denso de silicone de condensação para moldagem odontológica (Coltene).O perfil é composto basicamente por polidimetilsiloxano (PDMS), óleo mineral, carga inorgânica (possivelmente nano partículas de sílica), pigmento e aroma - para cada $1 \mathrm{~kg}$ de perfil denso, pode-se extrair aproximadamente $400 \mathrm{~mL}$ de PDMS; (PDMS-OH, Aldrich, tem preço aproximado de $\mathrm{R} \$$ 317,00 por $10 \mathrm{~mL}$, e o perfil denso, Coltene, aproximadamente $\mathrm{R} \$ 70,00$ a $\mathrm{R} \$ 83,00$ por $1 \mathrm{~kg}^{10}$ ).

É nítido que formulações com baixas concentrações de PDMS propiciam a fratura do composto, e que soluções com grandes concentrações tem gelificação quase que instantânea. A escolha da formulação ideal para aplicação deveria ter viscosidade adequada para a penetrabilidade nas amostras de pedra sabão (de acordo com a bibliografia consultada, optou-se por utilizar o viscosímetro de Ostwald visando encontrar viscosidade próxima à da água, entre 1,307 e 0,4665 $c P)$, sendo proposta a adição de aguarrás $\left(\mathrm{C}_{10} \mathrm{H}_{16}\right)$. PDMS e aguarrás passaram por mistura magnética por 15 minutos, seguida da adição de TEOS e agitação vigorosa por 2 minutos, mistura ultrassônica por 10 minutos, adição de DBTL catalisador dibutil estanho dilaurato [DBTL - 95\% Aldrich - $\left.\left(\mathrm{Ca}_{32} \mathrm{H}_{64} \mathrm{O}_{4} \mathrm{~S}\right)_{n}\right]$ - e novos 15 minutos de agitação ultrassônica. (Erro! Fonte de referência não encontrada. 7). Chegou-se a viscosidade de $\eta=$ 1,2460 cP, conforme a Equação 1:

Onde:

$$
\frac{\eta_{1}}{\eta_{2}}=\frac{d_{1} t_{1}}{d_{2} t_{2}}
$$

(1) $\eta_{1}$ é a viscosidade do líquido 1 , e consequentemente $\eta_{2}$ viscosidade do líquido 2

$\mathrm{d}_{1}$ é a densidade do líquido 1 , e $\mathrm{d}_{2}$ consequentemente do líquido 2

$t_{1}$ é o tempo de escoamento do líquido 1 , e consequentemente $t_{2}$ tempo de escoamento do líquido 2 (utilizou-se a água, com medidas sob condições normais de temperatura e pressão)

A impregnação foi realizada com pincéis, mantendo cada superfície úmida por 1 minuto, e tempo de secagem/gelificação de sete dias com base nos testes supracitados. Para as seis amostras de $7 \times 7 \times 7 \mathrm{~cm}^{3}$, o tempo de secagem entre as demãos foi de aproximadamente 20 minutos, com gasto de 
cerca de $80 \mathrm{~mL}$ de produto para todo o processo (5 demãos).

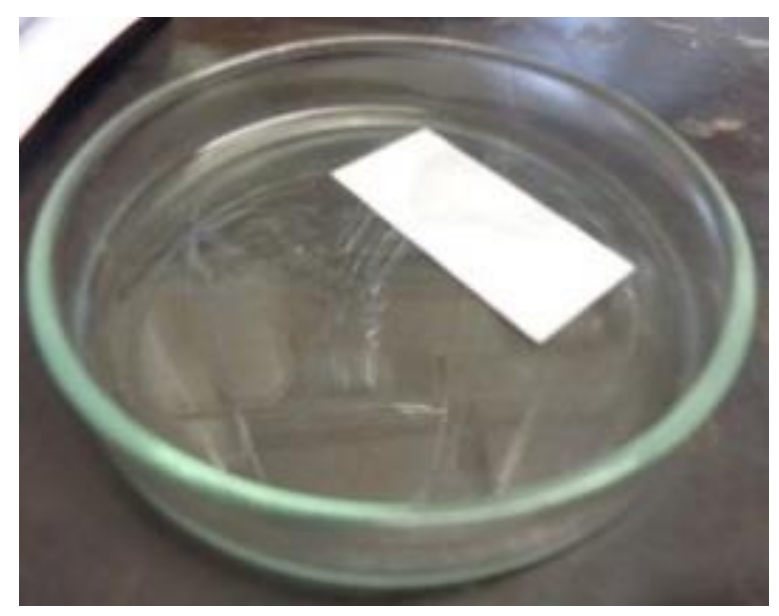

Figura 7. Consolidante Sol-gel híbrido, desenvolvido entre 16 a 28 de jan. 2015 (Elaboração dos autores)

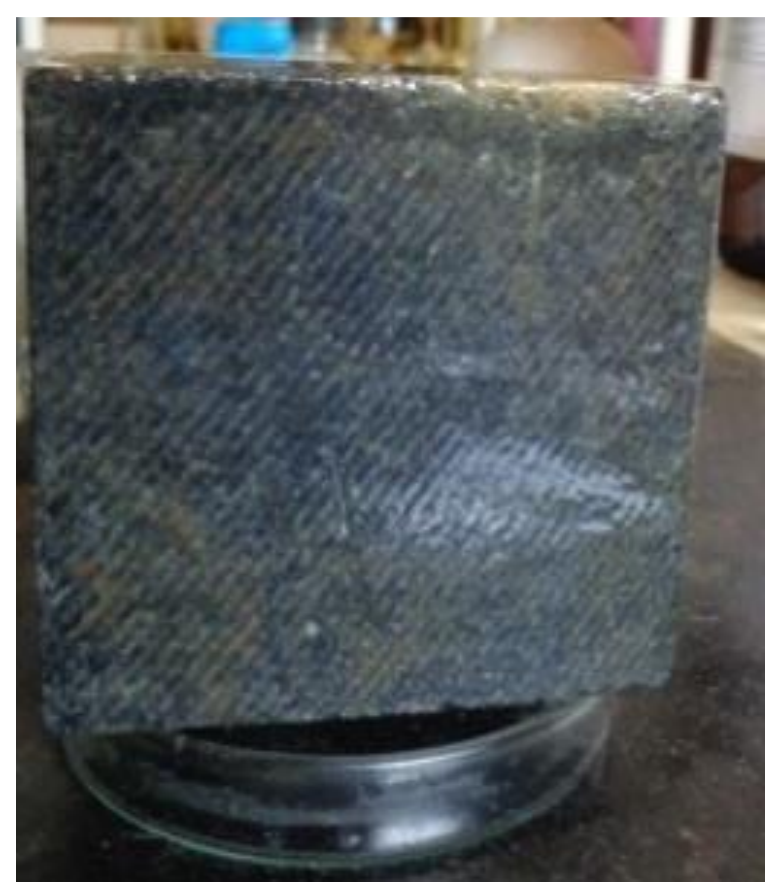

Figura 8. Aspecto após 1 ạ demão (Elaboração dos autores - fev. 2015)

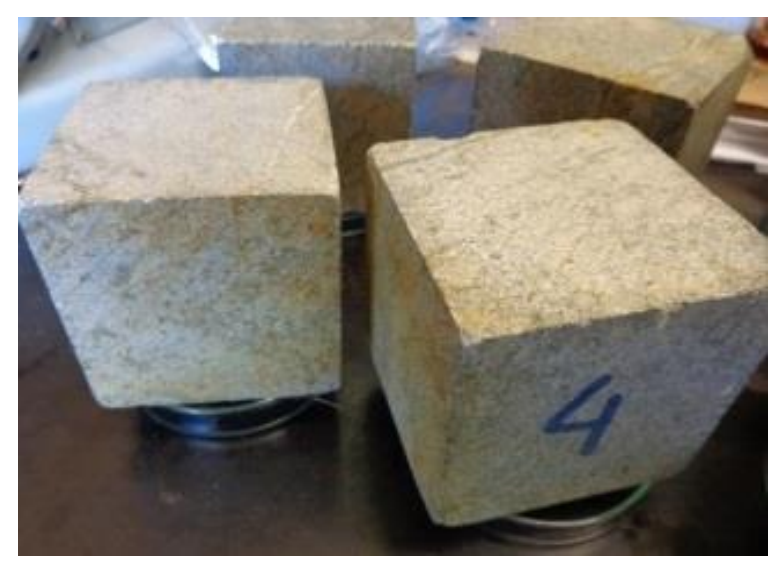

Figura 9. Aspecto após 5a demão (Elaboração dos autores - fev. 2015)

\section{APRESENTAÇÃO, ANÁLISE E DISCUSSÃO DOS RESULTADOS}

Ensaios auxiliaram na validação do produto proposto. O ensaio de determinação da densidade aparente, porosidade aparente e da absorção de água (ABNT/NBR 15.845/10), exibiu redução da porosidade aparente (de 0,38\% para 0,20\%) e da absorção da água (de 0,13\% para 0,07\%). A redução da porosidade faz referência direta à redução dos espaços vazios internos, esses associados às descontinuidades internas derivadas de processo de deterioração. A impregnação de consolidante atinge, portanto, o objetivo de preencher as diversas fraturas internas, ainda com ganho de resistência devido à redução da porosidade, e certa tendência hidrofóbica da mesma, visto a redução da absorção de água.

Tal resultado é corroborado pelo ensaio de determinação da resistência à compressão axial (ABNT/NBR 15.845/10) que conta como resultado a média de tensão de ruptura de 34,69 MPa para a amostra desgastada, e $36,96 \mathrm{MPa}$ para a consolidada. O ensaio de determinação de absorção de água por capilaridade (ABNT/NBR 9.779/1994) confirma a tendência hidrofóbica do composto testado, uma vez que há uma notável redução da absorção de água após consolidação. (GRÁFICO 1).

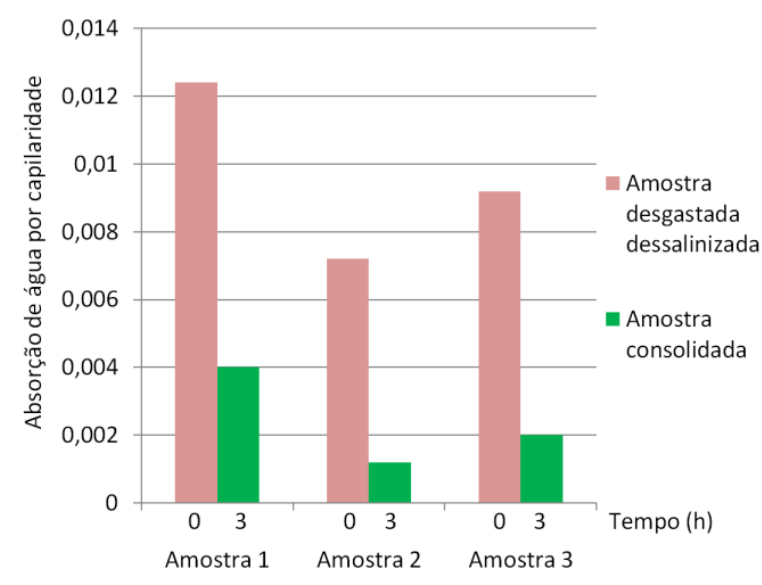

Gráfico 1. Gráfico comparativo da capilaridade para as amostras dessalinizadas e deterioradas, e após consolidação - peças de $5 \times 5 \times 5 \mathrm{~cm}^{3}$ (Elaboração dos autores - jul/2015)

É notado, por fim, que a presença de sais nos poros e capilares das amostras leva a uma redução das propriedades mecânicas do material, sendo necessário, portanto, a consolidação. O produto híbrido estudado serve ao objetivo inicial proposto de consolidar, visto que possibilita a melhora das características mecânicas das amostras testadas, devendo ocorrer discussões mais amplas e ensaios mais sistemáticos para garantia de funcionalidade do composto, bem como de sua estabilidade ao 
longo do tempo. Frisa-se a inalterabilidade da estética das peças.

\section{CONSIDERAÇÕES FINAIS}

Frente aos resultados apresentados nos ensaios realizados, pode-se argumentar que os sais podem entrar por capilaridade na pedra sabão. Uma vez com sais, a pedra sabão apresenta obstáculos para sua conservação. $\mathrm{O}$ sal pode ter inúmeras origens, como da própria parte mineral solúvel da pedra, do uso incorreto de materiais de intervenção, do transporte pelo ar (o que pode ocorrer com as estátuas em pedra sabão de Congonhas do Campo em Minas Gerais, relacionado com as mudanças da direção dos ventos e da presença de particulados referentes aos danos produzidos pela atividade mineratória), e ainda pelo metabolismo de microrganismos (esses que podem ser vinculados com os fungos liquenizados que contribuem também para a infiltração de água na rocha, favorecendo a deterioração da peça).

Frente a defasagem da base teórica atual, é indicada a realização dos ensaios relacionados à flexão, tração e deformabilidade; conhecimento de alguns outros parâmetros do esteatito, como módulo de elasticidade e demais resistências, e a caracterização química e física do consolidante. É necessária ainda a caracterização dos poluentes existentes especificamente na região em que o consolidante será aplicado, uma vez que a poluição contribui para a aceleração do processo de deterioração.

\section{REFERÊNCIAS BIBLIOGRÁFICAS}

AMERICAN SOCIETY FOR TESTING AND MATERIALS.ASTM D-2845: standard test method for laboratory determination of pulse velocities and ultrasonic elastic constants of rock. West Conshohocken, PA, USA: ASTM, 2000.

ANGELI, Matthieu. Multiscale study of stone decay by salt crystallization in porous networks: etude multiéchelle de la degradation des roches par la cristallisation de selsdans les réseauxporeux. 2007. Tese (Doutorado) - Geologia, Université de Cergy-Pontoise, Cergy-Pontoise, 2007.

ASSOCIAÇÃO BRASILEIRA DE NORMAS TÉCNICAS. NBR 15.845. Rio de Janeiro: ABNT, 2010.

CALLISTER, William D. Materials science and engineering: an introduction. 2 ed. New York: John Wiley \& Sons Inc, 1991.

CARMO, Joaquim Ribeiro do. Aleijadinho e a dança estática. Belo Horizonte: 1973.

CHAGAS, Mário. Museália. RJ, 1996.

COSTA, Antônio Gilberto. Rochas e Histórias do Patrimônio Cultural do Brasil e de Minas. Nacional, Bem te vi, 2009.

DENTRAL CREMER. Silicone de Condensação perfil - Coltene. $2016 . \quad$ Disponível em: < http://www.dentalcremer.com.br/produto/298451/siliconede-condensacao-perfil-denso-coltene100642>. Acessoem: set. 2016.
DOEHNE, Eric; PRICE, Clifford A. Stone Conservation: an overview of current research. 2 nd ed. Getty Publications.The Getty Conservation institute. Los Angeles, 2010.

EL-GOHARY, M. 2011.Chemical deterioration of egyptian limestone affected by saline water. Int.J. Cons. Sci. 2 (1) 1728.

ESPINOSA, R.M., FRANKE, L., DECKELMANN, G. 2008. Model for the mechanical stress due to the salt crystallization in porous materials. Constr. Build Mater. 22 (7) 1350-1367.

FIGUEIREDO JÚNIOR, João Cura D’Ars de. Química Aplicada à conservação e restauração de bens culturais: uma introdução. Belo Horizonte, São Jerônimo, 2012.

Handbook of SOL-GEL Science and Technology: Processing, Characterization and Applications. Edited by Sumio Sakka. Osaka, Japan: Kluwer Academic Pub, 2004.

HENCH, Larry L; WEST, Jon k.The Sol-gel process. Chem. Rev., Washington, v.90, n. 1, p. 33-72. (Revised Manuscript Received October 27, 1989).

HERKENRATH, Gudrum M; MARQUES, Adair E. IDEAS Investigations into devices against environmental attack on stones. Geesthacht, Germany: GKSS-Forschiengs-zentrum Geesthacht Gmbh, 1994, 237 p.

INSTITUTO DO PATRIMÔNIO E ARTísTICO NACIONAL IPHAN. Manual de conservação de cantarias. 2000. Disponivel em: <http://www.iphan.gov.br/baixaFcdAnexo.do;jsessionid=D96 C9655A052041E8894E82BEB98F23D?id=3091>. Acesso em: nov. 2013.

INSTITUTO PORTUGÊS DE QUALIDADE. NP EN 12.370: métodos de ensaio para pedra natural - determinação da resistência à cristalização de sais. Caparica, Portugal: IPQ, 2001.10 p.

KIM, Bun Kyung; WON, Jongok; DO, Jin-young; KIM, Sa Dug; KANG, Yong Soo. Effects of silica nanoparticle and GPTMS addition on TEOS-based stone consolidants. Elsevier: Journalof Cultural Heritage, Córeia, 2008. Disponível em: http://dx.doi.org/10.1016/j.culher.2008.07.008. Acesso em: 03 set. 2013.

LI, Dan; XU, Feigao; LIU, Zhenghua; ZHU, Jiaqi; ZHANG, Qingjian; $\mathrm{LI}$, Shao. The effect od adding $P D M S-O H$ and sílica nanoparticles on sol gel properties and effectiveness in stone protection. Elsevier: Applied Surface Science, China, 2012. Disponívelem:

http://dx.doi.org/10.1016/j.apsusc.2012.12.030. Acessoem: 04 fev. 2013.

LIU etal.The effect of adding PDMS-OH and silica nanoparticles on sol-gel properties and effectiveness in stone protection.2012.

OROSCO, E. As avarias nas esculturas do Período Colonial de Minas Gerais. Revista do Serviço do Patrimônio Histórico e Artístico Nacional, n. 5, p. 179-206, Rio de Janeiro. 1941.

ROBERT, Warley. Mestre Aleijadinho - Antônio Francisco Lisboa. [200-]. Disponível em: <http://www.congonhas.com.br/>. Acesso em: 01 jun. 2013.

SALAZAR-HERNÁNDEZ, Carmen; CERVANTES, Jorge; PUY-ALQUIZA, MaríaJesús; MIRANDA, Raúl. Conservation of building materials of historic monuments using a hybrid formulation.Journalof Cultural Heritage.V. 16, n.2, 2014. Disponivel em: <http://www.sciencedirect.com/science/article/pii/S1296207 414000533>. Acesso: jan. 2015.

SALAZAR-HERNÁNDEZ, Carmen; PUY-ALQUIZA, MaríaJesús; SALGADO. Patricia; CERVANTES, Jorge.TEOS-colloidal sílica PDMS-OH hybrid formulation used for stone consolidation. Appl. Organomental. Chem. Chichester, UK , v. 24, p. 481488. 2010. 
SIGMA-ALDRICH. Tetraethylorthosilicate. 2016. Disponível em: < http://www.sigmaaldrich.com/catalog/product/aldrich/1319 03 ?lang=pt\&region=BR $>$. Acesso em: set. 2016.

SILVA, Maria Elizabeth. Avaliação da susceptibilidade de rochas ornamentais e de revestimentos à deterioração - um enfoque a partir do estudo de monumentos do Barroco mineiro. 2007. 131 f. Tese (Doutorado) - Instituto de Geociências, Universidade Federal de Minas Gerais, Belo Horizonte, 2007.

STOREMYR, Per. Weathering of soapstone in a historical perspective. Elsevier: Materials characterization. Suiça, 2004.

TORRACA, G. The Scientist's Role in Historic Preservation with Particular Reference to Stone Preservation. In: Historical and Philosophical Issues in the Conservation of Cultural Heritage. Los Angeles; The Getty Conservation Institute, 1996. p. 439 450.

UNESCO. O patrimônio: legado do passado ao futuro. [200-]. Disponível <http://www.unesco.org/new/pt/brasilia/culture/worldheritage/heritage-legacy-from-past-to-the-future/>. Acessoem: jan. 2015.

WHEELER, George. Alkoxysilanesand the Consolidation of stone.1 ed. Los Angeles: Getty Conservation Institute, 2005.

ZÁRRAGA, Ramón; CERVANTES, Jorge; SALAZAR-HERNANDES, Carmen; WHEELER, George. Effect of the addition of hydroxyl-terminated polydimethylsiloxane to TEOS-based stone consolidants. Journal of Cultural Heritage, V.11, n.2, abril-jun, 2010. Disponível em: <http://dx.doi.org/10.1016/ j.culher.2009.07.002.> Acesso em: 03 jun. 2013.

ZHAO, Jing; LUO, Hongjie; WANG, Liqin; LI, Weidong; ZHOU, Tie; RONG, Bo. TEOS/PDMS-OH hybrid material for the consolidation of damaged pottery. In: INTERNATIONAL CONFERENCE ON MODERN CHEMICAL TECHNOLOGY IN THE PROTECTION OF CULTURAL HERITAGE, 2012, China. Heritage Science, 2013, p. 1-12. Disponível em: <http://www.heritagesciencejournal.com/content/1/1/12.> Acesso em: jan. 2014.

Contribuição ao 1‥ Simpósio Brasileiro de Caracterização e Conservação da Pedra 14 a 16 de dezembro de 2016, Congonhas - MG

Nota:

É de responsabilidade da comissão editorial do Simpósio a revisão gramatical, ortográfica, de citações e referências bibliográficas. As normas de submissão podem se diferenciar das desta revista. 